\title{
Evaluación de vitamina C por HPLC en el desarrollo postcosecha del tomate (Solanum lycopersicum v. Dominator)
}

\section{Evaluation of vitamin C postharvest tomato (Solanum lycopersicum $v$. Dominator) by HPLC}

\author{
Manuel Elías Valle Colchao y Gilbert Rodríguez Paúcar \\ Departamento de Ingeniería Agroindustrial, Universidad Nacional del Santa
}

\section{RESUMEN}

La investigación tuvo como finalidad evaluar el desarrollo fisiológico postcosecha del tomate procedente del Valle de Santa, Áncash. Se cosechó en madurez fisiológica, analizando el tomate hasta su madurez sensorial. Se ha evaluado variación del contenido de vitamina C, pérdida de peso, sólidos solubles, $\mathrm{pH}$, acidez titulable, índice de madurez, índice de respiración, color y humedad. Se ha establecido que el contenido de vitamina $C(\mathrm{mg} / 100 \mathrm{gr})$ disminuye desde $85 \pm 0,04$ (estadío 1) hasta 26 $\pm 0,03$ (estadío 7 ). También desde el primer día hasta el último los resultados en función a su peso disminuye en $5,79 \%$, los sólidos

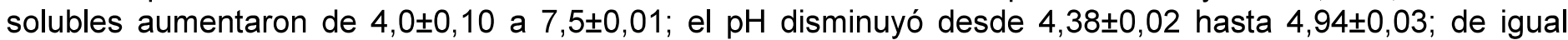
manera su acidez titulable $(6,45 \% \pm 0,02 \%-2,69 \% \pm 0,01 \%)$; el índice de respiración inmediatamente después de ser cosechada aumenta significativamente de 40,42 $\pm 0,10$ hasta $71,12 \pm 0,96$ en el estadío 6 , mientras que en el estadío 7 disminuye a $64,33 \pm 0,11$ y el color del tomate, notándose un color verde a rojo total.

Descriptores: tomate, dominator, vitamina C, postcosecha, CLAR

\section{ABSTRACT}

Research was intended to evaluator tomato development postharvest comes from the Valley of Santa, Ancash. It was harvested in physiological maturity, analyzing the tomato up to his sensory maturity. The evaluations were content of vitamin $\mathrm{C}$, lost weigh, solid solubles, $\mathrm{pH}$, acidity, maturity rate, respiration rate, color, humidity. At the end of the analysis, is established that the vitamin $C(\mathrm{mg} / 100 \mathrm{gr})$ content decreases from $85 \pm 0,04$ ( 1 stage), to $26 \pm 0,03$ ( 7 stage),. Whereas from the first to the last day, the results its weight diminishes in $5,79 \%$, soluble solid increases from $4,0 \pm 0,10$ to $7,5 \pm 0,01$; pH decreases from $4,38 \pm 0,02$ to $4,94 \pm 0,03$; acidity decreases $(6,45 \% \pm 0,02 \%-2,69 \% \pm 0,01 \%)$; the rate respiration immediately after being harvested increases significantly from $40,42 \pm 0,10$ to $71,12 \pm 0,96$ ( 6 stage), whereas in the 7 stage diminishes $64,33 \pm 0,11$ and the color, being green color to red total.

Keywords: tomate, dominator, vitamin C, postharvests, HPLC

\section{INTRODUCCIÓN}

El valle del Santa es unos de las principales zonas agrícolas de la provincia ya que se cosechan gran parte de frutas y hortalizas, siendo una de ellas el tomate, pues en el año 2007 la superficie cosechada fue de 101 hectáreas [1] y esto se debe al uso de plantines que consiste en mayor aprovechamiento de la semilla.

Por tal razón se consideró como referencia para el análisis de su desarrollo fisiológico postcosecha, así de mismo modo investigar el desarrollo de los demás productos de la provincia, de tal forma obtener un buen material bibliográfica. $Y$ esta información ayude a tomar decisiones en la aplicación de programas postcosecha para facilitar un mejor manejo, almacenamiento, conservación, empacado y transporte.

La Vitamina $C$ es un nutrimento esencial para los humanos. Una baja ingesta causa una enfermedad, por deficiencia, conocida como escorbuto. Esta Vitamina está presente en forma natural en muchas frutas y verduras $[2,3]$.

Una de las principales características durante el desarrollo fisiológico del tomate son los cambios bioquímicos, como la pérdida de nutrientes, en este caso la Vitamina C. Pues esta vitamina como en toda fruta y hortaliza, varía dependiendo del grado 
de madurez, aumenta su cantidad mientras están en la planta, luego tiende a disminuir desde el inicio de su cosecha; por lo que el tomate maduro pierde parte del contenido de vitamina C $[4,5]$.

La determinación de Vitamina $\mathrm{C}$ ha sido ampliamente estudiada por diferentes técnicas analíticas, teniendo en cuenta las propiedades fisicoquímicas de la molécula. Sin embargo la Cromatografía Líquida de Alta Resolución (HPLC), garantiza límites de detección y cuantificación más bajos, que facilita además la eliminación de los efectos causados por la matriz (interferencias en otros métodos de análisis); esta técnica es utilizada frecuentemente en investigaciones agroindustriales, bioquímica y química analítica $[6,7,8,9]$.

El objetivo principal de este estudio, fue cuantificar el contenido de Vitamina C por HPLC durante el desarrollo postcosecha del tomate. A la vez evaluar pérdida peso, sólidos solubles, $\mathrm{pH}$, acidez, humedad, respiración, color.

\section{EXPERIMENTAL}

Se utilizó tomate de la variedad Domintator, del Valle de Santa. Se recolectó en estado de madurez fisiológica; etapa donde el tomate ha alcanzado su máximo desarrollo y peso, además, ha adquirido el color verde característico; posteriormente se seleccionó y clasificó uniformemente. Se analizó hasta su madurez sensorial.

La cuantificación de vitamina $C$ se realizó usando un HPLC - Shimadzu, modelo LC-10AT, con los siguientes accesorios: Columna CLC-C18 SHIMPACK de Diámetro Interno 4,6 mm, Longitud: 150 $\mathrm{mm}$, Tamaño de partícula: $5 \mu \mathrm{m}$. Detector de Arreglo de Diodos $(245 \mathrm{~nm})$. Autosampler (inyector automático).

\section{Curva de calibración de Vitamina $\mathbf{C}$}

Para la obtención de la curva se usó Ácido Ascórbico, QP, preparando concentraciones de 0.2 , $0.4,0.6$ y $0.8 \mathrm{mg} / \mathrm{mL}$, se acondicionó el equipo, Tabla 1, obteniendo cromatogramas y finalmente la curva de calibración, ver gráfico 1.

Tabla 1: Condiciones de Trabajo

\begin{tabular}{|l|l|l|}
\hline \multirow{2}{*}{ Fase Móvil } & Metanol & $65 \%$ \\
\cline { 2 - 3 } & Agua Filtrada & $35 \%$ \\
\hline Tasa de Flujo & 1.0 & $\mathrm{~mL} / \mathrm{min}$ \\
\hline Temperatura & 40.0 & ${ }^{\circ} \mathrm{C}$ \\
\hline Volumen de Muestra & 5 & $\mathrm{uL}$ \\
\hline
\end{tabular}

\section{\begin{tabular}{|l|l}
\hline Patrón de Muestra & Ac. Ascórbico \\
\hline
\end{tabular}}

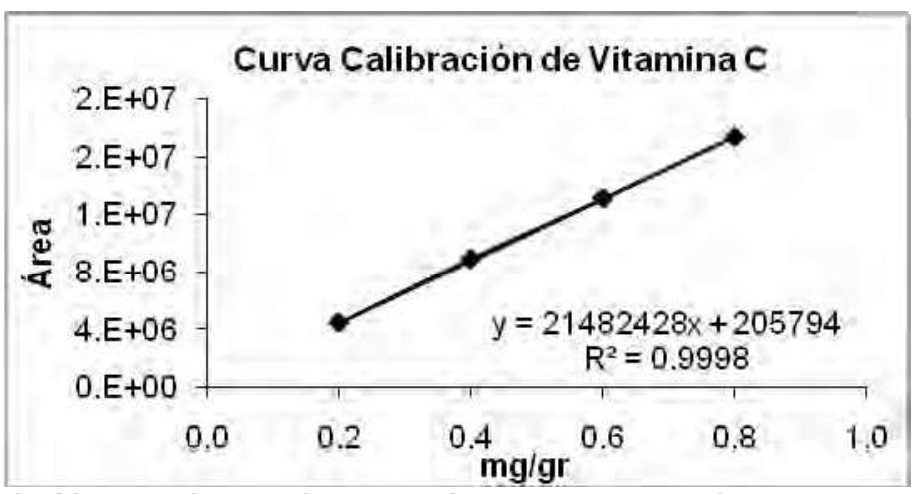

Gráfico 1: Curva Calibración de Vitamina C Análisis de Vitamina $\mathrm{C}$ del Tomate

Se extrajo el jugo del tomate, se pesó $5 \mathrm{gr}$, luego se adicionó $15 \mathrm{~mL}$ de orto fosfórico al $4.5 \%$, posteriormente se adicionó $25 \mathrm{ml}$ agua ultra pura; se llevo a la centrifuga a 4000 r.p.m. por $15 \mathrm{~min}$; al término se filtró en Discos de Porosidad de 0.20 um; finalmente se analizó en el HPLC y se reemplazó en la Curva de Calibración.

\section{Análisis del Desarrollo Fisiológico}

La determinación del contenido de sólidos solubles se realizó con un refractómetro (marca: Hand. Held Atago), haciendo la lectura de ${ }^{\circ} \mathrm{Brix}$ directamente del equipo. Para la Acidez Titulable (\%Ácido Cítrico) se determinó en base al método 10.026 de la AOAC.

$\mathrm{El} \mathrm{pH}$ se determinó con un potenciómetro digital (marca: Shott Gerate), por inmersión del electrodo en el jugo del tomate previa calibración con solución buffer de $\mathrm{pH} 4$ y 7 a $25^{\circ} \mathrm{C}$.

Para el índice de Respiración, se utilizó un respirómetro. Con respecto al color se midió mediante un colorímetro C400 (kónica Minolta Sensing INC, Japón). La humedad se determinó por secado y diferencia de pesos de acuerdo al método 934.06 (37.1.10) del AOAC.

\section{RESULTADOS Y DISCUSIONES}

Evaluación de Vitamina C (mg/100gr) en el desarrollo postcosecha 


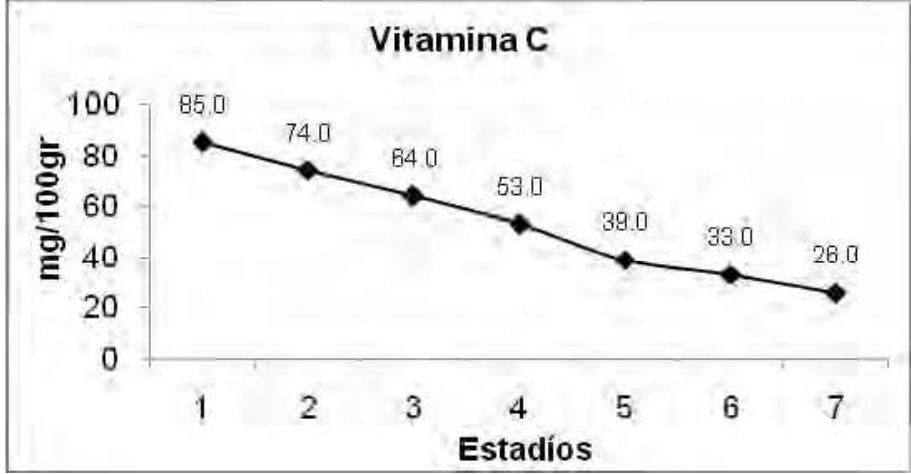

Gráfico 2: Evaluación de Vitamina C (mg/100gr)

Después de la cosecha de frutas y hortalizas sufren cambios bioquímicos como los carbohidratos, pigmentos, vitaminas, minerales, proteínas, sabor, textura, aroma, apariencia [5]. Existen numerosos ejemplos de la influencia genética en el contenido de vitaminas. Es más fácil encontrar una información similar relativa a los efectos del grado de madurez. Sin embargo, los tomates son un ejemplo excelente; varía el contenido de ácido ascórbico con el grado de madurez pues es máximo cuando está todavía verde [10]. Una de las principales fuentes de vitamina $C$ es el camu camu con $2780 \mathrm{mg} / 100 \mathrm{~g}$, comparada con la naranja, el camu camu proporciona 30 veces más vitamina $C$ [11], otras fuentes son las hortalizas como es el caso de la Col Silvestre $136 \mathrm{mg} / 100 \mathrm{~g}$ [12]. Los requerimientos diarios de Vitamina C según la Organización Mundial de la Salud (OMS) recomiendan 45 miligramos [13]. En el gráfico 2, notamos que el contenido de vitamina $C$ del tomate en su madurez sensorial es de $26 \mathrm{mg} / 100 \mathrm{~g}$, de tal forma si deseamos completar la dieta diaria de Vitamina C sugerida por OMS tendríamos que consumir $100 \mathrm{gr}$., aproximadamente 2 a 3 tomates diarios, lo cual sería difícil para una persona normal. Pero al aplicar ciertos programas postcosecha, se podría conservar el contenido de vitamina $\mathrm{C}$, y así la dosis diaria de tomate sería menor.

\section{Evaluación de Sólidos Solubles ( ${ }^{\circ}$ Brix) en el desarrollo postcosecha}

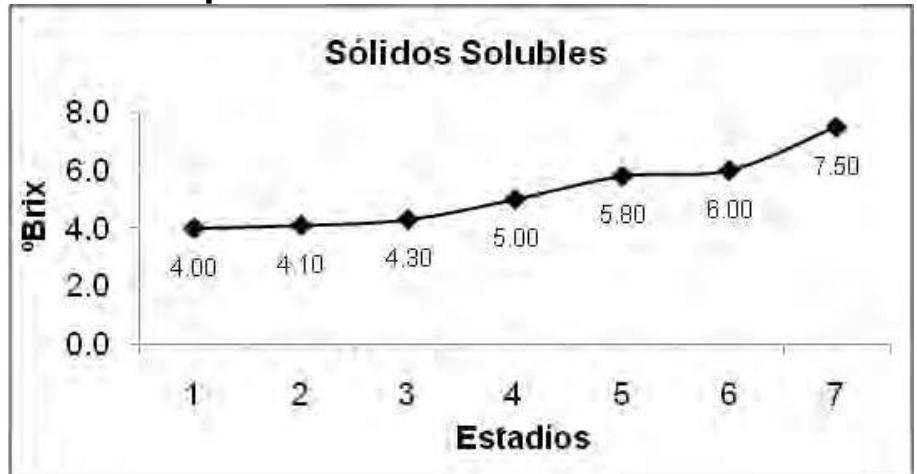

Gráfico 3: Evaluación de Sólidos Solubles ( ${ }^{\circ}$ Brix)
Evaluación de pH y Acidez Titulable (\%Ác. Cítrico) en el desarrollo postcosecha

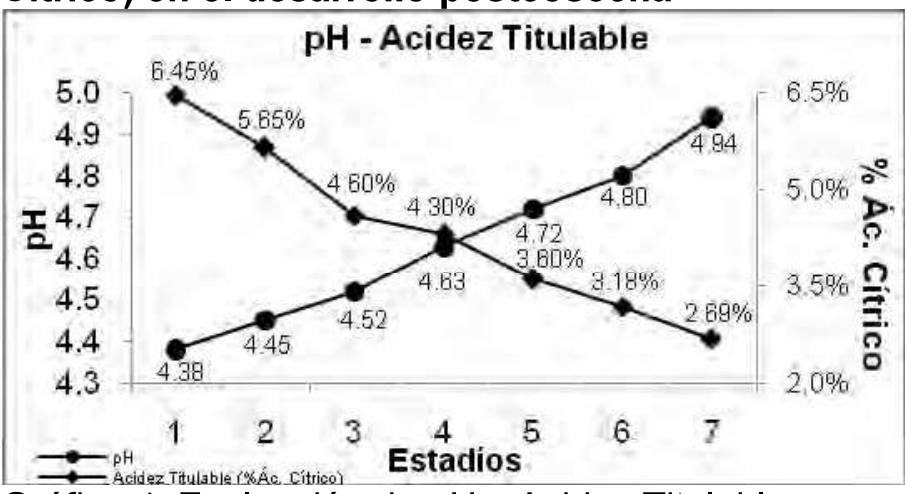

Gráfico 4: Evaluación de $\mathrm{pH}$ y Acidez Titulable

Los hidratos de carbono sufren cambios bioquímicos durante la maduración. La degradación de los polisacáridos de las membranas celulares, ejercen una contribución Importante sobre el aumento en contenido de azucares [14]. Los ácidos cítricos son los más frecuentes y abundantes en tejidos de plantas comestibles. En la mayoría de las frutas, el contenido de ácidos orgánicos disminuyen durante y después del proceso de maduración [15]. La tendencia de los sólidos solubles y del $\mathrm{pH}$, durante los días postcosecha fue ascendente, mientras la acidez titulable descendió. Los ${ }^{\circ}$ Brix varían de 4,0 para luego aumentar hasta $7,5^{\circ}$ Brix, estadío 7 , como se observa en el Gráfico 3.

Mientras que el pH varía de 4,49. De manera inversa sucede con la Acidez Titulable ya que de $6,45 \%$ desciende hasta $2,69 \%$, ver Gráfico 4.

\section{Evaluación de Índice de Madurez ('Brix/\%Acidez) en el desarrollo postcosecha}

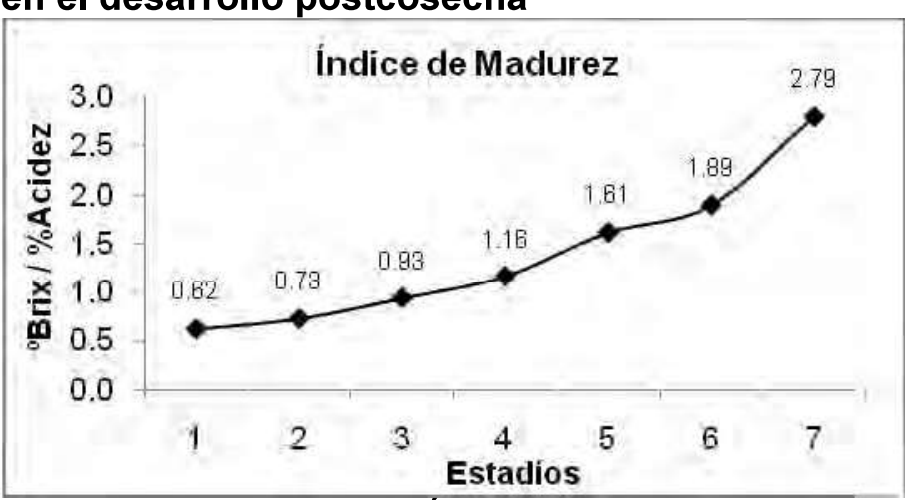

Gráfico 5: Evaluación de Índice de Madurez

La medida del grado de madurez de las frutas. Es la relación de grados Brix/acidez. El índice aumenta con la maduración, ya que se incrementa los sólidos solubles y disminuye la acidez [5]. La variación del Índice de Madurez se incrementa de 0,62 (estadío 1) hasta 2,79 (estadío 7); entre el 6 y 7 existe un aumento considerablemente, como se muestra en el gráfico 5 . 
Evaluación de Índice de Respiración $\mathrm{CO}_{2} / \mathrm{Kg} . \mathrm{Hr}$ ) en el desarrollo postcosecha

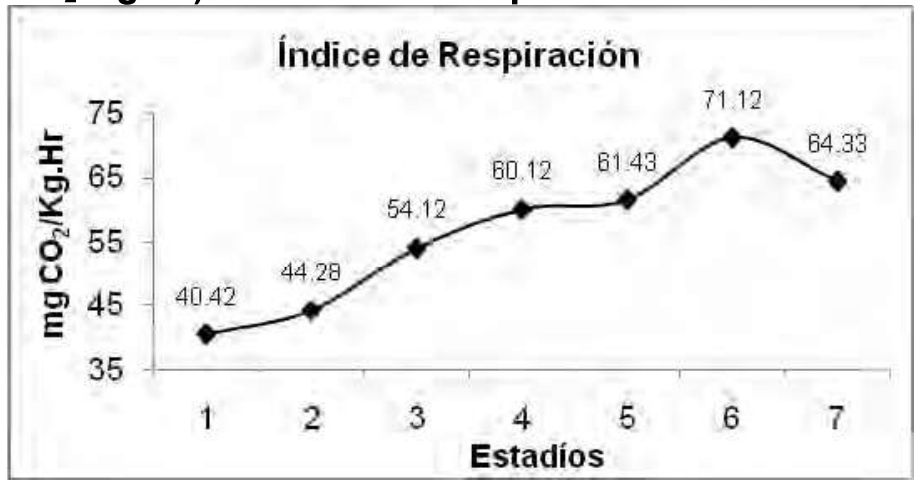

Gráfico 6: Evaluación de Índice de Respiración

Durante la maduración, existe un tremendo aumento en la cantidad de etileno producido. Este aumento usualmente es acompañado por un aumento en la tasa de respiración de la fruta (un fenómeno llamado el climaterio). La tasa de respiración y de producción de etileno usualmente depende de la temperatura de almacenamiento, edad de la fruta y del cultivar/híbrido [15]. En la gráfica 6 se puede apreciar un aumento de respiración ya que se trata de un fruto climatérico; pero en el estadío 7 disminuye. Al sexto estadío se presenta el mayor valor de respiración $(71,12 \mathrm{mg} \mathrm{CO} / \mathrm{kg} \cdot \mathrm{hr}-0,36$ $\mathrm{Kcal} / \mathrm{kg} . \mathrm{hr}$ ). El Índice promedio de Respiración fue $56,55 \mathrm{mg} \mathrm{CO} / \mathrm{kg} \cdot \mathrm{hr}-0,29 \mathrm{Kcal} / \mathrm{kg} \cdot \mathrm{hr}$.

Evaluación del color en el desarrollo postcosecha

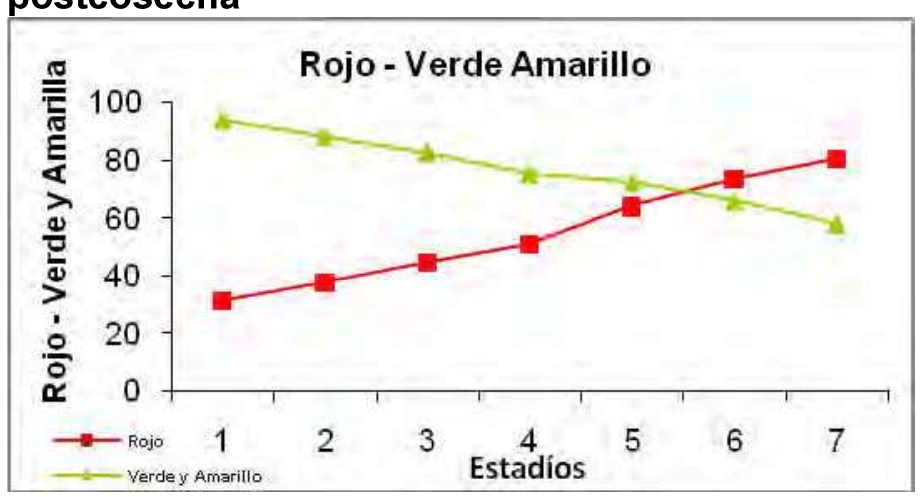

Gráfico 7: Evaluación de Tono Rojo, Verde Amarillo

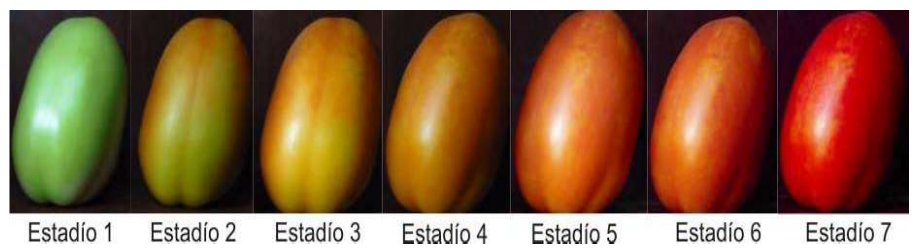

Figura 1: Variación de Color

El color del tomate, verde al principio y rojo cuando madura, se debe a la degradación de clorofila en los cromoplastos de las células y síntesis de carotenos, entre los que destaca el licopeno. Como consecuencia de ello, el parámetro $a^{*}$ cambia de valores negativos (color verde) a positivos (color rojo) [5]. En el estadío 1; se observa que el producto esta totalmente de color verdes es por eso que en el gráfico 7 el tono rojo es mucho mayor al verde amarillo; y así va variando los tono en forma inversa; tanto así que el final en el estadío 7; el tono rojo es mucho mayor que el tono verde.

Evaluación de Materia Seca y Humedad en el desarrollo postcosecha

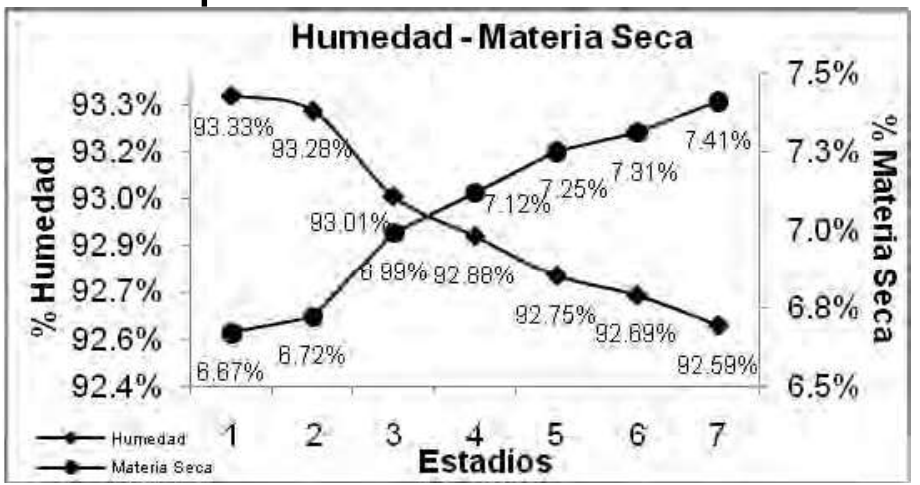

Gráfico 8: Evaluación de Materia Seca y Humedad

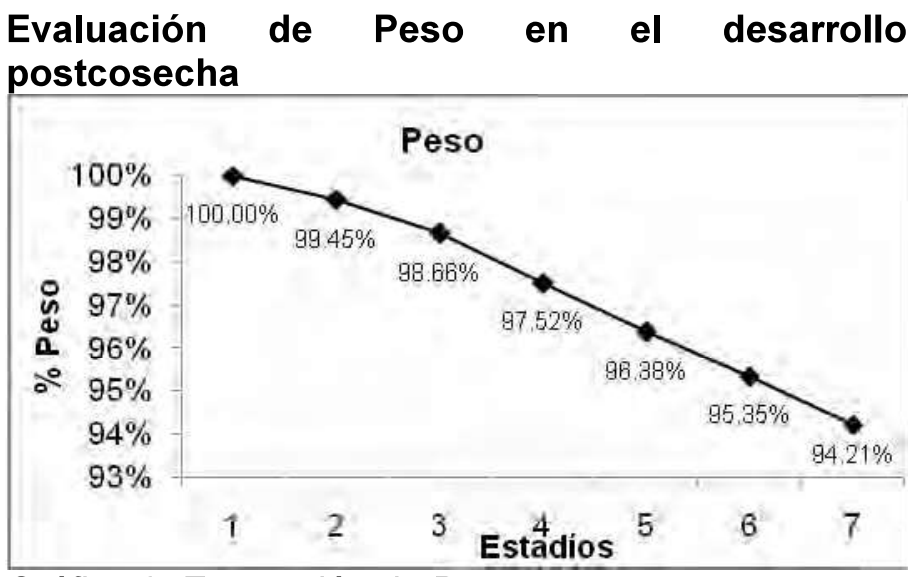

Gráfico 9: Evaluación de Peso

El agua se pierde al ambiente como vapor de agua moviéndose desde los espacios intercelulares existentes en el parénquima hacia la atmósfera exterior, la liberación puede darse a través de las lenticelas, las estomas o la cutícula [16]. Con los valores obtenidos en el laboratorio y mostrados en el gráfico 9 , se puede deducir que la pérdida de peso del tomate es uniforme. Se considera que pierde $5,79 \%$ de su peso desde el primer hasta su madurez sensorial.

\section{Cromatogramas de Vitamina C}




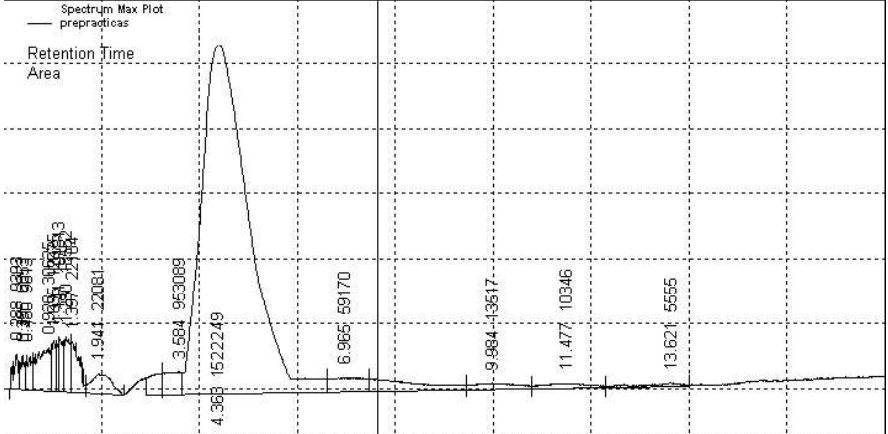

Figura 3: Estadío 1

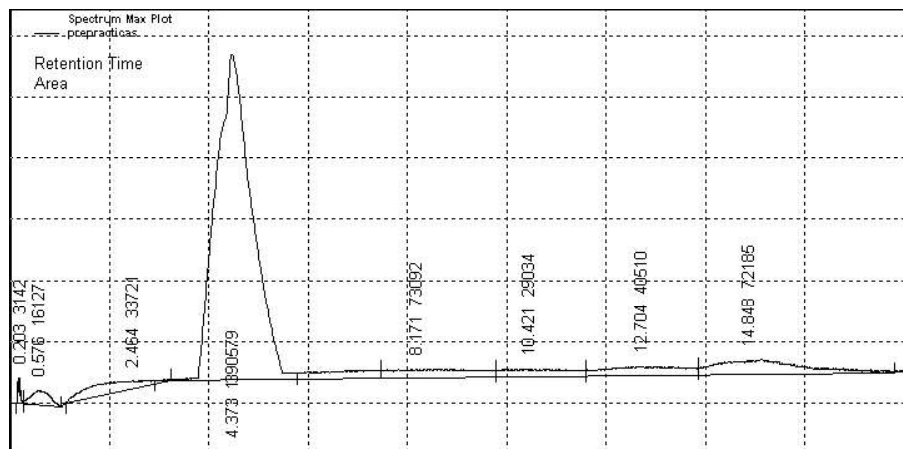

Figura 4: Estadío 2

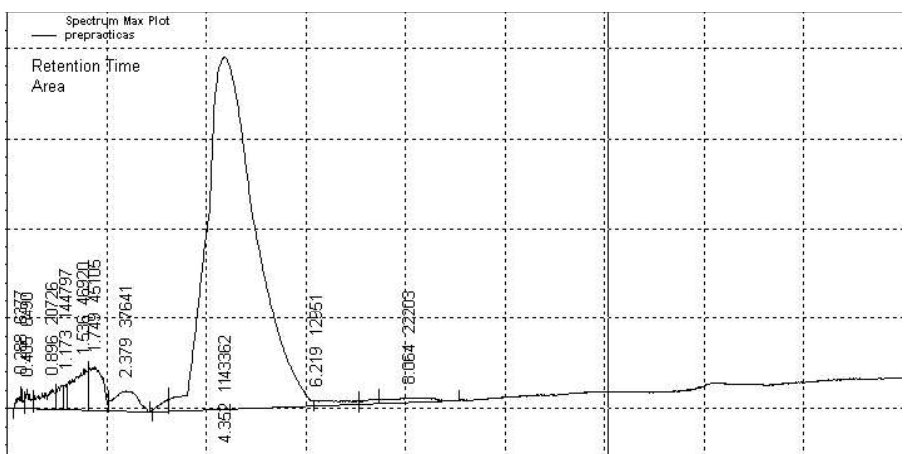

Figura 5: Estadío 3

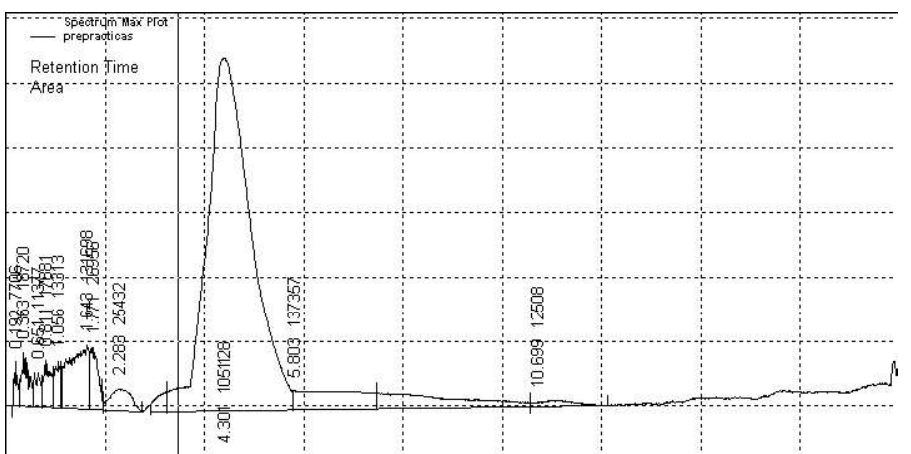

Figura 6: Estadío 4

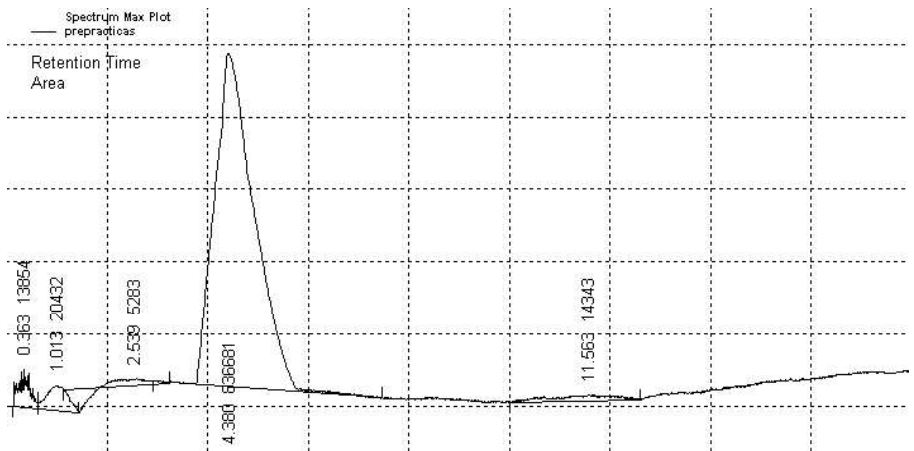

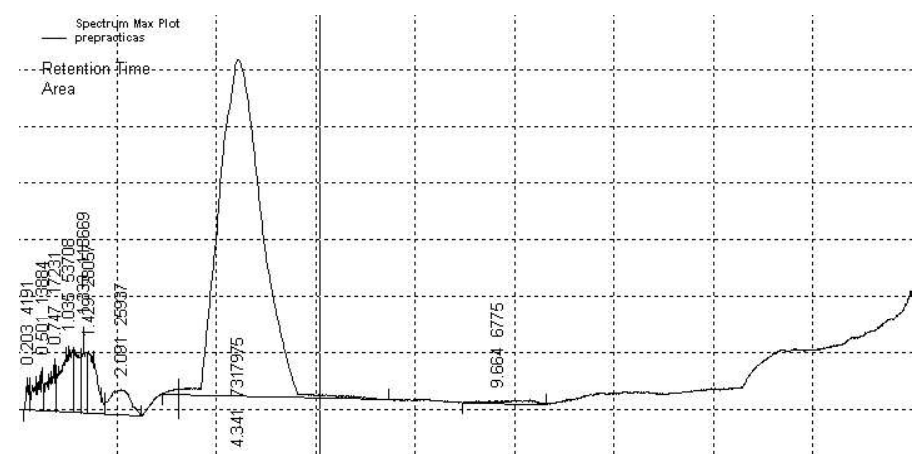

Figura 8: Estadío 6

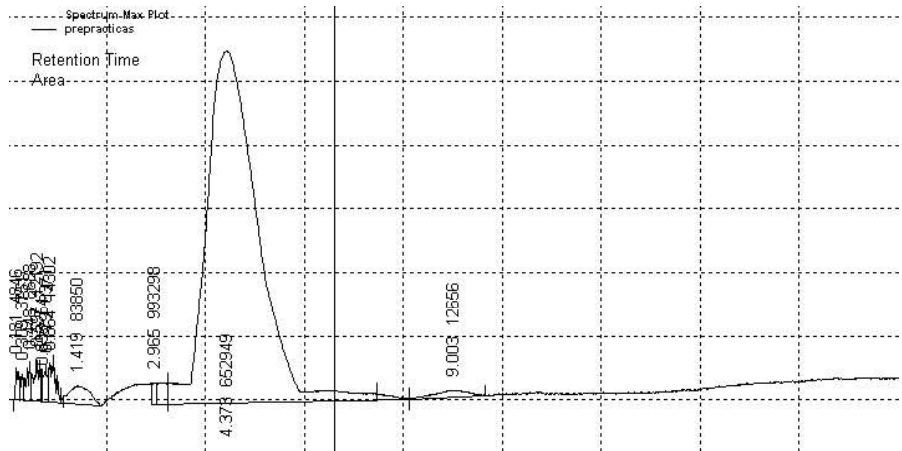

Figura 9: Estadío 7

En los cromatogramas, Figuras de arriba, se nota la aparición de un pico que por el tiempo de retención (4.36 $\mathrm{min}$ ) se considera la presencia de Vitamina C, y también, a su lado, se observa su respectiva área.

\section{CONCLUSIONES}

El tomate, después de cosechada continúa respirando, madurando y en algunos casos iniciando el procesos de senescencia, todo lo cual implica una serie de cambios como Pérdida de Peso, Sólidos Solubles, pH, Acidez, Humedad, Respiración, Vitaminas, Color; que son determinados para cada fruta.

El contenido de vitamina C disminuye considerablemente en un $70 \%$, esto se debe a que el tomate es un producto climatérico y por ende después de su cosecha utiliza sus reservas como los carbohidratos y los ácidos orgánicos hasta su senescencia; pues esto es unos de los factores que degrada la vitamina $\mathrm{C}$.

Aplicando alguna Tecnología Postcosecha como por ejemplo Tipo de Empaque y Temperatura de Almacenamiento, se podría lograr una buena conservación nos sólo del contenido de vitamina C sino también de los demás nutrientes del tomate.

\section{AGRADECIMIENTO}

Al Jefe MSc. Gilbert Rodríguez Paúcar y Técnica Ing. Soledad Quezada Berrú de Laboratorio de Investigación y Desarrollo de Productos

Figura 7: Estadío 5 
Agroindustriales de la Universidad Nacional del Santa; por su asesoramiento incondicional.

\section{REFERENCIAS}

[1] Ministerio de Agricultura de la República de Perú (2008).

[2] N. Block, y M. Hudes, American Journal of Epidemiology (2001).

[3] Z. Jhon, y R. Yudkin, The Lancet, 359; (2002) 1969-1974.

[4] FAO, Manual de Manejo postocoseha de frutas tropicales (papaya, piña, plátano y cítrico) 2000.

[5] R. P. Gilbert, Manual de Práctica de Laboratorio de Fisiología y Tecnología Postcosecha (2009).

[6] M. Thin, y M. FARID, Ultraviolet treatment of orange juice, Innovate Food Science and Emerging Technologies, 5; (2004) 495-502.

[7] K. GIANNA, Food Chemistry, 83 (2003) 33-41.

[8] A. RIZZOLO, y A. BRAMBILLA, Evaluation of sampling and extraction procedures for the analysis of ascorbic acid from pear fruit tissue. Food Chemistry, 77; (2002) 257-262.

[9] O. Quattrocchi, et, Introducción a la HPLC Aplicación y Práctica, Artes Gráficas Farro SA, Argentina (1992).

[10] O. R. Fennema, Química de los Alimentos, 2da. Edición (2000).

[11] CEDECAM Centro de Desarrollo para la Competitividad de la Amazonía, Tratado de Cooperación Amazónica (2010).

[12] Instituto Nacional de Salud, Tablas Peruanas de Composición de Alimentos, Ministerio de Salud, 8va. Edición (2009).

[13] World Health Organization, Vitamin and mineral requirements in human nutrition (2004).

[14] R. G. Carlos, Manejo Postcosecha y Evaluación de la Calidad de Tomate (Lycopersicum esculetum Mill) que se comercializa en la Ciudad de Neiva, Universidad Sur Colombiana (1998).

[15] A. A. Kader, Respiration and gas exchange of vegetables, Marcel Dekker Inc., New York, (1987).

[16] Sena, Manual de Fisiología, Reino Unido (1995).

E-mail: manuelvallecolchao@hotmail.com giropape@yahoo.com 